\title{
Radiology in the Era of Big Data and Machine Learning
}

\author{
Augusto BF Antunes ${ }^{1}$, Flávio Amaro Oliveira Bitar Silva ${ }^{2 *}$ and Guilherme Augusto Salgado ${ }^{3}$ \\ ${ }^{1}$ Radiologist at Axial Medicina Diagnóstica, Brazil \\ ${ }^{2}$ Trauma and Urgency Surgeon; Gastrointestinal Endoscopist, Brazil \\ ${ }^{3}$ Medical Consultant and Head of Business Development, Brazil
}

Submission: June 10, 2017; Published: Jun 16, 2017

*Corresponding author: Flávio Amaro Oliveira Bitar Silva, General Surgeon, Trauma and Urgency Surgeon, Gastrointestinal Endoscopist, Medical Consultant at Kunumi, Rua Trifana 247/901 CEP: 30210-570, Belo Horizonte, Minas Gerais (MG), Brazil, Email: flavioamaro.obs@gmail.com

\section{Abstract}

The whole healthcare system, from management to care providing, including scientific production, will be impacted by Artificial Intelligent. Nowadays, uniting data availability, powerful hardwares and new Deep Learning algorithms, there is a perfect environment to really develop a new way of solve problems in healthcare practice. Radiology has always been intrinsically connected to new scientifical advances and technologies, probably like no other area in medicine. It's time to consider what the best place for the radiologist is, in this context. Also, it is imperative to know that there is no way to really ingress on this new era of technology based development in healthcare, if no effort is to be made on organizing, store, retrieve, distribute and secure the data we produce.

Keywords: Artificial Intelligence; Machine Learning; Deep Learning; Healthcare future; Radiology.

\section{Opinion}

Thought leaders worldwide believe that we are going through the Fourth Industrial Revolution, when new technologies will integrate the physical, the digital and the biological worlds, with an immense impact in a wide variety of disciplines, industries and markets [1].

Since its inception in 1895, when Wilhelm Röntgen discovered the x-ray, through its "golden decade" in the 1970s, with the first Computed Tomography (CT) scanners devised by Godfrey Hounsfield and Allan Cormack and Magnetic Resonance Imaging (MRI) studies by Paul Lauterbur and Peter Mansfield, Radiology has been intrinsically connected to new scientific advances and technologies, probably like no other area in medicine.

Radiology is facing another period of dramatic changes, mainly driven by the amount of medical data that grows in an exponential pace and advances in Artificial Intelligence (AI), which have the potential to completely remodel the field and the role of radiologists [2].

Big Data is the term given to datasets that are incredible large, with sizes and complexity beyond the ability of traditional data processing applications to deal with. Data is everywhere, generated by increasing numbers of mobile devices, computers and sensors that gather information on the environment and human interactions, ranging from weather patterns to medical records. To put things in perspective, in 2013 the amount of data generated and stored encompassed 4.4 zettabytes (1021). In 2020 , this number is estimated to rise to 44 zettabytes [3].

Machine Learning (ML) is a subfield of AI that gives "computers the ability to learn without being explicitly programmed" [4]. Extremely complex data, such as that generated by the healthcare system or even one large scale hospital, are unpredictable, being next to impossible for a programmer or even an specialist to consider every scenario and handle them properly. ML shifts this burden to the algorithm, which is capable of identifying patterns and relationships in data beyond human capacity, allowing it to find correlations and make predictions with variable levels of sensitivity and accuracy.

Computer-Aided Diagnosis (CAD) powered by ML algorithms are a powerful tool, being able to help in interpreting imaging findings, reducing interpretation times and especially in minimizing errors or misinterpretation. As radiologists, we rely heavily on pattern recognition while interpreting medical images and coming up with possible diagnoses. In fact, as demonstrated by an ellegant study using Functional Magnetic Ressonance 
Imaging (fMRI), we generate diagnostic hypotheses fast and efficiently by neurocognitive mechanisms used in non-analytical reasoning tasks, such as naming objects or concepts in everyday life [5].

Multiple algorithms have been used for different and challenging tasks in Medical Imaging [6-8], delivering robust results. Applications can range from CAD, image segmentation and content-based retrieval, to text analysis of radiology reports using natural language processing (NLP). ML for Radiology is already here, not some vague possibility in a distant future.

One problem that ML technology is facing is the quality of the data available. There is plenty of datasets out there, but their quality don't match the requirements of state-of-the-art techniques. Not only data collected on hospitals or companies is of lower standards, but also datasets that were systematically acquired by careful designed protocols, such as those of big initiatives for collecting medical data, like the Alzheimer's Disease Neuroimaging Initiative (ADNI)[9]. These brilliant initiatives were conceived with the premise that many diseases are to big a challenge to be tackled by only a few groups of investigators, often apart from each other.

We are currently working on a research using the ADNI dataset and some ML techniques, especially Deep Learning and convolutional neural networks (CNN), trying to predict Alzheimer's Dementia progression. These neural networks are able to create computational models that are composed of multiple processing layers that learn representations of data with higher levels of abstraction [10], being especially effective in object recognition and localization in images. Currently, we are processing standardized imagens through the model. It's a laborious step, but what took the most of our time, was analyzing ADNI's dataset. Working with this dataset is not simple as it could be. Even with their rigorous methods of collection, data was not stored and organized adequately for a straightaway study using CNNs, requiring some effort to treat it satisfactory, besides the usual problems that are expected with big datasets, like missing data, misleading labels, etc.

Thus, it becomes imperative for anyone involved with Medical Research or Healthcare to reconsider some aspects of data, not only its quantity or the quality, but also how organized it will be stored, how efficiently it can be retrieved and distributed and how secure it is guarded. Radiology departments, hospitals, scientific institutions and companies need to evaluate these aspects and maybe re-think their relationship with data, even redesigning structurally in order to achieve an efficient and rapid use of data, one that matches the immense capabilities of the current technology and is capable of unleashing all its benefits for patients and society.

Another point that is shaping radiology concerns the direct relationship between ML and physicians. Radiologists should not fear these new advances in technology, but embrace them instead. While some analyses can be automated, others cannot. There will be a displacement of our functions, roles will be redefined, but that change is as natural as any that can come with the development of new technologies. We will need to adapt to $\mathrm{AI}$, leaving mechanical and time-consuming tasks to machines, while dedicating to more cognitively demanding tasks and providing for every patient the "human touch" that robots are incapable of.

The interaction between humans and $\mathrm{AI}$ is often tricky to predict, but ML is already here to enhance our potential as radiologists. Also, it would be wise to say that we should keep our friends close and AI even closer.

\section{Conflict of Interest}

The authors work on Kunumi (www.kunumi.ai), a Brazilian startup company dedicated on bring innovative solutions to complicated problems, through its Machine Learning and Deep Learning proprietary models. Kunumi has a Health Division, responsible for applying those models to science production and to solve healthcare problems.

\section{References}

1. https://www.weforum.org/agenda/2016/01/the-fourth-industrialrevolution-what-it-means-and-how-to-respond/

2. Jha S, Topol E (2016) Adapting to Artificial Intelligence-Radiologists and Pathologists as Information Specialists. JAMA 316(22): 23532354.

3. http://medicalfuturist.com/artificial-intelligence-will-redesignhealthcare/

4. Samuel A (1959) Some Studies in Machine Learning Using the Game of Checkers. IBM Journal 3(3): 535-554.

5. Melo M, Scarpin DJ, Amaro E, Passos RB, Sato JR, et al. (2011) How doctors generate diagnostic hypotheses: a study of radiological diagnosis with functional magnetic resonance imaging. PLoS One 6(12): e28752.

6. Wang S, Summers RM (2012) Machine Learning and radiology. Med Image Anal 16(5).

7. Greenspan H, Van Ginneken B, Summers RM (2016) Deep Learning in Medical Imaging: Overview and Future Promise of an Exciting New Technique IEEE Transctions on Medical Imaging 35(5).

8. Erickson (2017) Machine Learning for Medical Imaging. Radiographics $37(2)$.

9. http://adni.loni.usc.edu

10. Le Cun Y, Bengio Y, Hinton G (2015) Deep learning. Nature volume 521. 
(C) This work is licensed under Creative

(CC) DOI: 10.19080/CTCMI.2017.01.555575
Your next submission with Juniper Publishers will reach you the below assets

- Quality Editorial service

- Swift Peer Review

- Reprints availability

- E-prints Service

- Manuscript Podcast for convenient understanding

- Global attainment for your research

- Manuscript accessibility in different formats

( Pdf, E-pub, Full Text, Audio)

- Unceasing customer service

Track the below URL for one-step submission https://juniperpublishers.com/online-submission.php 